\title{
Effect of Modified Roux-en-Y Gastric Bypass Surgery on GLP-1, GIP in Patients with Type 2 Diabetes Mellitus
}

\author{
Shao-Wei Xiong, ${ }^{1}$ Jing Cao, ${ }^{1}$ Xian-Ming Liu, ${ }^{1}$ Xing-Ming Deng, \\ Zeng Liu, ${ }^{1}$ and Fang-Ting Zhang ${ }^{2}$ \\ ${ }^{1}$ Department of General Surgery, Peking University Shenzhen Hospital, Shenzhen, Guangdong 518036, China \\ ${ }^{2}$ Central Laboratory, Peking University Shenzhen Hospital, Shenzhen, Guangdong 518036, China \\ Correspondence should be addressed to Fang-Ting Zhang; fangtingzhang@126.com
}

Received 24 April 2015; Revised 5 June 2015; Accepted 10 June 2015

Academic Editor: Daniele Marrelli

Copyright (C) 2015 Shao-Wei Xiong et al. This is an open access article distributed under the Creative Commons Attribution License, which permits unrestricted use, distribution, and reproduction in any medium, provided the original work is properly cited.

The type 2 diabetes mellitus (T2DM) is one of the most serious diseases that threaten public health. Modified gastric bypass surgery has been applied to the treatment of T2DM patients in the 1990s, but the therapeutic mechanism to this function is still unclear. The aim of this study was to further clarify the effect and the mechanism of modified gastric bypass surgery on glucose metabolism in patients with T2DM. In the study, the incretin indexes and blood glucose indexes were analyzed before surgery and 1 week and 1,3 , and 6 months after surgery. The results suggested that modified Roux-en-Y gastric bypass can promote GLP-1 secretion in patients with T2DM, while reducing the secretion of GIP. Thus it could effectively control blood glucose of patients with T2DM.

\section{Introduction}

The type 2 diabetes mellitus (T2DM) is a kind of commonly and frequently encountered disease which severely threatens people's health. Completely cured T2DM is a difficult problem on medicine. Modified gastric bypass surgery began to be used in T2DM patients in the 1990s [1,2], and the efficiency of this procedure reached $83 \%$ [3-10] although the therapeutic mechanism involved is still unclear. In order to further clarify the effect and the mechanism of modified gastric bypass surgery on glucose metabolism in patients with T2DM, we retrospectively analyzed incretin indexes including GLP-1, GIP, and some blood glucose indexes in patients with T2DM underwent modified Roux-en-Y gastric bypass procedures.

\section{Materials and Methods}

2.1. General Data. A retrospective analysis of the clinical data of 50 patients with T2DM and gastric cancer or simple T2DM treated in the department of general surgery from January 2006 to September 2014 was conducted. The patients underwent gastrectomy using modified Roux-enY gastric bypass procedures. Follow-up data were complete.
The patients in this study were diagnosed with T2DM in accordance with the diagnostic criteria of the American Diabetes Association (2009). Specific parameters measured were as follows: symptoms of diabetes mellitus, random blood glucose $\geq 11.1 \mathrm{mmol} / \mathrm{L}$, fasting plasma glucose $\geq 7.0 \mathrm{mmol} / \mathrm{L}$, and an oral glucose tolerance test at $2 \mathrm{~h} \geq 11.1 \mathrm{mmol} / \mathrm{L}$. If the patients met one of the above conditions and retest the following day conformed to the standards of the diagnosis of diabetes, the patient was then diagnosed with diabetes mellitus. Islet cell antibodies, antibody to insulin, glutamic acid decarboxylase antibody, glycosylated hemoglobin (HbAlc), and $C$ peptide level were examined to define T2DM.

In 50 patients, 27 patients were males, the rest 23 are female, the average age is $46.18+11.36$ years (the age range is $30-66$ years), and the mean BMI is $25.23+1.21 \mathrm{~kg} /$ square meters.

2.2. Surgery. Patients with T2DM and gastric cancer were treated with radical resection of gastric cancer firstly. According to the location of cancer in the gastric cavity, the patients underwent radical total gastrectomy or distal gastrectomy (residual gastric cavity volume $30-50 \mathrm{ML}$ ), stomach perigastric lymph node dissection. Simple T2DM patients were 
TABLE 1: Comparison of glucose metabolism index before and after the surgery.

\begin{tabular}{lccccc}
\hline & $n$ & BMI & FBG & 2hPBG & HbAlc \\
\hline $0 \mathrm{mo}$ & 50 & $25.23 \pm 1.21$ & $10.3 \pm 1.1$ & $14.1 \pm 2.8$ & $9.8 \pm 1.1$ \\
$1 \mathrm{wk}$ & 50 & $24.82 \pm 1.16$ & $9.2 \pm 1.3$ & $13.2 \pm 2.5$ & $9.7 \pm 1.2$ \\
$1 \mathrm{mo}$ & 50 & $24.35 \pm 1.27$ & $6.5 \pm 1.5^{\#}$ & $8.1 \pm 1.9^{\#}$ & $9.0 \pm 1.1$ \\
$3 \mathrm{mo}$ & 50 & $24.03 \pm 1.15$ & $5.5 \pm 1.4^{\#}$ & $6.2 \pm 1.5^{\#}$ & $6.7 \pm 1.3^{*}$ \\
$6 \mathrm{mo}$ & 50 & $23.87 \pm 1.22$ & $5.0 \pm 1.1^{\#}$ & $6.5 \pm 1.8^{\#}$ & $5.1 \pm 1.2^{\#}$ \\
\hline
\end{tabular}

Data are shown as mean $\pm \mathrm{SD}, \mathrm{BMI}\left(\mathrm{kg} / \mathrm{m}^{2}\right), \mathrm{FBG}$, and $2 \mathrm{hPBG}(\mathrm{mmol} / \mathrm{L})$, HbAlc (\%).

Compared with the before surgery ( 0 months) group ${ }^{*} P<0.05,{ }^{\#} P<0.01$.

treated with cutting transversely in the upper gastric body to close the gastric cavity, residual gastric cavity volume $30-$ $50 \mathrm{ML}$. And then all patients were treated with modified Roux-en-Y gastric bypass procedures. The jejunum was cut $75-100 \mathrm{~cm}$ from the Treitz ligament, and then the distal end of the jejunum was connected to the residual stomach or esophagus by an end-to-end or end-to-side anastomosis. The distal jejunum at $75-100 \mathrm{~cm}$ was connected to the proximal jejunum by an end-to-side anastomosis.

2.3. Observations. Body mass index (BMI), fasting blood glucose (FBG), two-hour postprandial blood glucose (2hPBG), hemoglobin Alc (HbAlc), fasting glucagon-like peptide-1 (GLP-1), two-hour glucagon-like peptide-1 (2hGLP-1), fasting gastric inhibitory polypeptide (FGIP), and two-hour gastric inhibitory polypeptide (2hGIP) levels were determined before surgery ( 0 months) and 1 week and 1, 3, and 6 months after surgery, respectively.

2.4. Statistical Analysis. The measurement data were expressed as the mean \pm SD. Statistical analyses were performed by the $t$ test using SPSS17.0 (SPSS Inc., Chicago, IL, USA). $P<0.05$ was considered statistically significant.

\section{Results}

Compared with the preoperative FBG and 2hPBG levels, these levels decreased 1,3 , and 6 months after surgery $(P<$ $0.01)$. HbAlc levels were significantly decreased 3 and 6 months after surgery $(P<0.05)$. FGLP-1 and 2hGLP-1 levels were significantly increased at each time point after surgery $(P<0.05)$. Compared to FGLP-1 levels, the levels of 2hGLP-1 were significantly higher at the same time point $(P<0.01)$. FGIP levels were significantly decreased 1,3 , and 6 months after surgery $(P<0.05)$ and 2 hGIP levels were significantly decreased at each time point after surgery $(P<0.01)$. Compared to FGIP levels at the same time point, the levels of 2 hGIP were significantly higher before surgery $(P<0.01)$, but no significant differences were found between the two groups after surgery (Tables 1 and 2, Figures 1 and 2).

\section{Discussion}

Type 2 diabetes is a chronic metabolic syndrome marked by high levels of glucose in the blood. Owing to its various etiological factors and complicated mechanisms, the traditional
TABLE 2: Comparison of GLP-1 and GIP levels before and after the surgery.

\begin{tabular}{lccccc}
\hline & $n$ & FGLP-1 & 2hGLP-1 & FGIP & 2hGIP \\
\hline $0 \mathrm{mo}$ & 50 & $6.5 \pm 1.3$ & $11.9 \pm 1.6^{\#}$ & $347 \pm 16$ & $801 \pm 21^{\#}$ \\
$1 \mathrm{wk}$ & 50 & $9.3 \pm 1.1^{*}$ & $19.7 \pm 2.0^{\mathbf{\star}}$ & $328 \pm 15$ & $436 \pm 25^{\mathbf{\Delta}}$ \\
$1 \mathrm{mo}$ & 50 & $11.6 \pm 1.7^{\mathbf{\Delta}}$ & $23.7 \pm 1.9^{\mathbf{\#}}$ & $229 \pm 16^{*}$ & $238 \pm 17^{\mathbf{\Delta}}$ \\
$3 \mathrm{mo}$ & 50 & $17.9 \pm 1.9^{\mathbf{\Delta}}$ & $28.9 \pm 2.1^{\mathbf{\Delta}}$ & $217 \pm 12^{*}$ & $241 \pm 15^{\mathbf{\Delta}}$ \\
$6 \mathrm{mo}$ & 50 & $18.1 \pm 1.8^{\mathbf{\Delta}}$ & $29.2 \pm 2.2^{\mathbf{\Delta}}$ & $213 \pm 14^{*}$ & $230 \pm 13^{\mathbf{\Delta}}$ \\
\hline
\end{tabular}

Data are shown as mean \pm SD, FGLP-1 and 2hFGLP-1 (pmol/L), FGIP, and 2hFGIP (pg/ML).

Compared with the before surgery ( 0 months) group ${ }^{*} P<0.05,{ }^{\mathbf{\Lambda}} P<0.01$. Comparison of the same point in time ${ }^{\#} P<0.01$.

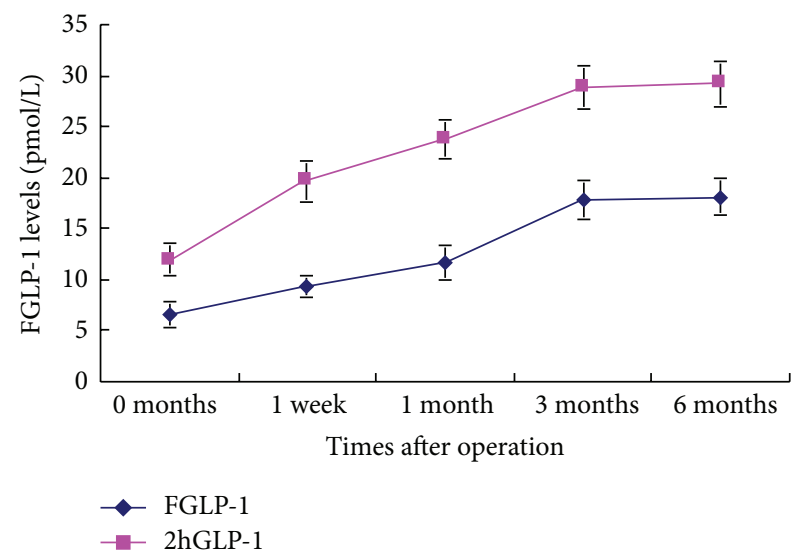

FIGURE 1: FGLP-1 levels before and after the surgery.

way of treatment to control blood glucose is lack of stability, unable to suppress the disease development fundamentally; therefore, curing T2DM has become an urgent global medical goal [11].

Modified gastric bypass surgery began to be used in T2DM patients in the 1990s, and the efficiency of this procedure to cure obese patients with T2DM is between 86.6 and $91 \%$ [3-10]; previous research showed that $83 \%-86 \%$ of T2DM patients maintained normal levels of blood glucose following operation $[12,13]$. In a follow-up study, American Diabetes Association [14] showed that this surgical procedure was also efficacious in T2DM patients without obesity.

In the present study, we retrospectively analyzed blood glucose indexes in patients with T2DM and gastric cancer or simple T2DM underwent gastrectomy using modified Roux-en-Y gastric bypass procedures. We found that, compared with the levels before surgery, FBG and $2 \mathrm{hPBG}$ levels were significantly decreased 1, 3, and 6 months after surgery; HbAlc levels were significantly decreased 3 and 6 months after surgery. The metabolism of blood glucose index improved obviously; therefore, the modified digestive tract reconstruction can better control blood glucose in patients with T2DM, and the curative effect is exact and reliable. Although the modified gastric bypass surgery had a better hypoglycemic effect and lasted longer [15], the mechanism is not very clear. 


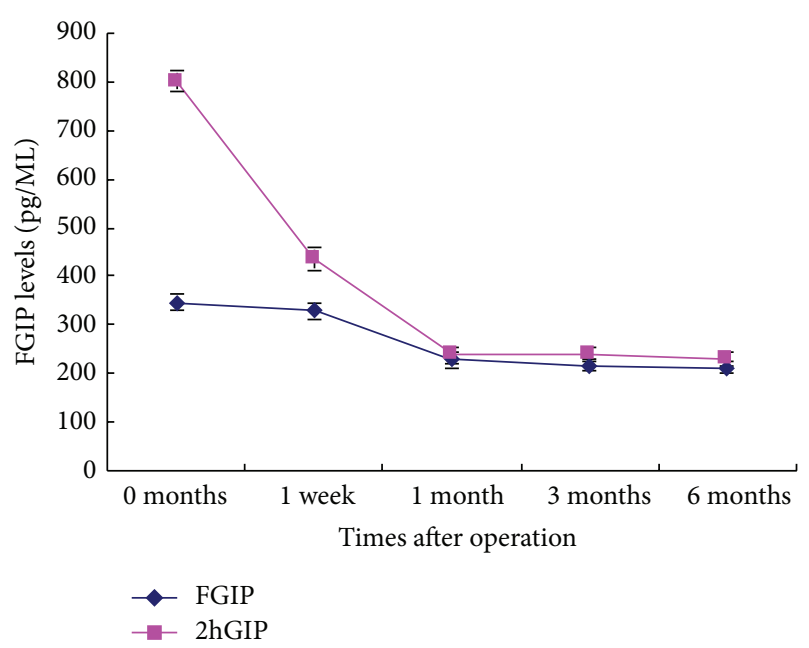

FIGURE 2: FGIP levels before and after the surgery.

At present, there are many hypotheses related to this mechanism, among which, "the foregut hypothesis" and "hindgut hypothesis" have received significant attention [16]. The "foregut hypothesis" considers that food in the duodenum and proximal jejunum stimulate the production of GIP, thus improving insulin resistance [17-19]. The "hindgut hypothesis" considers that chyme in the terminal ileum and colon promotes the secretion of GLP-1 and stimulates the secretion of glucose-dependent insulin, which improves the insulin effect and the proliferation of islet B cells [20-26].

In order to further clarify the effect of modified gastric bypass surgery on incretin secretion in patients with T2DM, we analyzed FGLP-1, 2hGLP-1, FGIP, and 2hGIP levels in patients with T2DM. We found that FGLP-1 and 2hGLP-1 levels were significantly increased at each time point after surgery. The level of 2 hGLP-1 was significantly higher than the level of FGLP-1 at the same time point. This is consistent with the changes in the secretion of GLP-1 in the "hindgut hypothesis."

Meanwhile, FGIP levels were significantly decreased 1, 3, and 6 months after surgery; 2hGIP levels were significantly decreased at each time point after surgery. Compared to FGIP levels at the same time point, the levels of $2 \mathrm{hGIP}$ were significantly higher before surgery, but no significant differences were found between the two groups after surgery. These data prove what the "the foregut hypothesis" said that food into the foregut stimulates the production of GIP. Following the modified digestive tract reconstruction procedure, the food does not enter the foregut, directly into the hindgut, and led to a decline in FGIP and 2hGIP levels. Therefore, modified Roux-en-Y gastric bypass surgery can promote GLP-1 secretion in patients with T2DM, while reducing the secretion of GIP, thus resulting in better regulation of blood glucose [27].

\section{Conflict of Interests}

The authors guarantee that there exists no conflict of interests in the paper.

\section{Authors' Contribution}

Shao-Wei Xiong designed the research; Shao-Wei Xiong, Xian-Ming Liu, and Zeng Liu performed the research; ShaoWei Xiong, Jing Cao, and Xing-Ming Deng analyzed data and wrote the paper; and Fang-Ting Zhang revised the paper.

\section{Acknowledgment}

This work is supported by The Basic Research Projects of Shenzhen R\&D Funding in Science and Technology, no. JCYJ20130402113802233 and no. JC201005260209A.

\section{References}

[1] M. L. Moanţă, M. Moța, C. Copăescu, and C. Dumitrache, "Effects of sleeve gastrectomy versus gastric bypass on type 2 diabetes mellitus remission in obese patients," Romanian Journal of Diabetes Nutrition and Metabolic Diseases, vol. 19, no. 4, pp. 361-371, 2012.

[2] H. Buchwald, R. Estok, K. Fahrbach et al., "Weight and type 2 diabetes after bariatric surgery: systematic review and metaanalysis," American Journal of Medicine, vol. 122, no. 3, pp. 248.e5-256.e5, 2009.

[3] K. Yip, L. Heinberg, V. Giegerich, P. R. Schauer, and S. R. Kashyap, "Equivalent weight loss with marked metabolic benefit observed in a matched cohort with and without type 2 diabetes 12 months following gastric bypass surgery," Obesity Surgery, vol. 22, no. 11, pp. 1723-1729, 2012.

[4] G. Mingrone, S. Panunzi, A. de Gaetano et al., "Bariatric surgery versus conventional medical therapy for type 2 diabetes," The New England Journal of Medicine, vol. 366, no. 17, pp. 1577-1585, 2012.

[5] W. J. Lee, K. Y. Hur, M. Lakadawala, K. Kasama, S. K. Wong, and Y. Lee, "Gastrointestinal metabolic surgery for the treatment of diabetic patients: a multi-institutional international study," Journal of Gastrointestinal Surgery, vol. 16, no. 1, pp. 45-52, 2012.

[6] P. P. Raj, R. Kumaravel, C. Chandramaliteeswaran, V. Vaithiswaran, and C. Palanivelu, "Laparoscopic duodenojejunal bypass with sleeve gastrectomy: preliminary results of a prospective series from India," Surgical Endoscopy, vol. 26, no. 3, pp. 688-692, 2012

[7] J. Reoch, S. Mottillo, A. Shimony et al., "Safety of laparoscopic vs open bariatric surgery: a systematic review and meta-analysis," Archives of Surgery, vol. 146, no. 11, pp. 1314-1322, 2011.

[8] M. Hirao, S. Takiguchi, H. Imamura et al., "Comparison of Billroth i and Roux-en-Y reconstruction after distal gastrectomy for gastric cancer: one-year postoperative effects assessed by a multi-institutional RCT," Annals of Surgical Oncology, vol. 20, no. 5, pp. 1591-1597, 2013.

[9] T. Sano and T. Aiko, "New Japanese classifications and treatment guidelines for gastric cancer: revision concepts and major revised points," Gastric Cancer, vol. 14, no. 2, pp. 97-100, 2011.

[10] I. Songun, H. Putter, E. M.-K. Kranenbarg, M. Sasako, and C. J. van de Velde, "Surgical treatment of gastric cancer: 15-year follow-up results of the randomised nationwide Dutch D1D2 trial," The Lancet Oncology, vol. 11, no. 5, pp. 439-449, 2010.

[11] M. Spanou and K. Tziomalos, "Bariatric surgery as a treatment option in patients with type 2 diabetes mellitus," World Journal of Diabetes, vol. 4, no. 2, pp. 14-18, 2013.

[12] P. R. Schauer, S. R. Kashyap, K. Wolski et al., "Bariatric surgery versus intensive medical therapy in obese patients with 
diabetes," The New England Journal of Medicine, vol. 366, no. 17, pp. 1567-1576, 2012.

[13] W. J. Lee, K. Chong, K. H. Ser et al., "Gastric bypass vs sleeve gastrectomy for type 2 diabetes mellitus: a randomized controlled trial," Archives of Surgery, vol. 146, no. 2, pp. 143-148, 2011.

[14] American Diabetes Association, "Standards of medical care in diabetes-2013," Diabetes Care, vol. 36, supplement 1, pp. S11S66, 2013.

[15] H. C. Lee, M. K. Kim, H. S. Kwon, E. Kim, and K.-H. Song, "Early changes in incretin secretion after laparoscopic duodenal-jejunal bypass surgery in type 2 diabetic patients," Obesity Surgery, vol. 20, no. 11, pp. 1530-1535, 2010.

[16] M.-C. Chen, Y.-C. Lee, W.-J. Lee, H.-L. Liu, and K.-H. Ser, "Diet behavior and low hemoglobin level after laparoscopic minigastric bypass surgery," Hepato-Gastroenterology, vol. 59, no. 120, pp. 2530-2532, 2012.

[17] D. W. Porter, N. Irwin, P. R. Flatt, C. Hölscher, and V. A. Gault, "Prolonged GIP receptor activation improves cognitive function, hippocampal synaptic plasticity and glucose homeostasis in high-fat fed mice," European Journal of Pharmacology, vol. 650, no. 2-3, pp. 688-693, 2011.

[18] R. S. Rao and S. Kini, "GIP and bariatric surgery," Obesity Surgery, vol. 21, no. 2, pp. 244-252, 2011.

[19] B. Abdelmalak, M. Ibrahim, J.-P. Yared, M. B. Modic, and C. Nasr, "Perioperative glycemic management in insulin pump patients undergoing noncardiac surgery," Current Pharmaceutical Design, vol. 18, no. 38, pp. 6204-6214, 2012.

[20] Y. Kitahara, K. Miura, R. Yasuda, H. Kawanabe, S. Ogawa, and Y. Eto, "Nateglinide stimulates glucagon-like peptide-1 release by human intestinal L cells via a K(ATP) channel-independent mechanism," Biological and Pharmaceutical Bulletin, vol. 34, pp. 671-676, 2011.

[21] M. Bose, B. Oliván, J. Teixeira, F. X. Pi-Sunyer, and B. Laferrère, "Do incretins play a role in the remission of type 2 diabetes after gastric bypass surgery: what are the evidence?” Obesity Surgery, vol. 19, no. 2, pp. 217-229, 2009.

[22] B. Laferrère, S. Heshka, K. Wang et al., "Incretin levels and effect are markedly enhanced 1 month after Roux-en-Y gastric bypass surgery in obese patients with type 2 diabetes," Diabetes Care, vol. 30, no. 7, pp. 1709-1716, 2007.

[23] M. L. Vetter, S. Cardillo, M. R. Rickels, and N. Iqbal, "Narrative review: effect of bariatric surgery on type 2 diabetes mellitus," Annals of Internal Medicine, vol. 150, no. 2, pp. 94-103, 2009.

[24] V. R. Aroda, R. R. Henry, J. Han et al., "Efficacy of GLP-1 receptor agonists and DPP-4 inhibitors: meta-analysis and systematic review," Clinical Therapeutics, vol. 34, no. 6, pp. 1247.e22-1258.e22, 2012.

[25] Y. J. Park, Z. Ao, T. J. Kieffer et al., "The glucagon-like peptide-1 receptor agonist exenatide restores impaired pro-islet amyloid polypeptide processing in cultured human islets: implications in type 2 diabetes and islet transplantation," Diabetologia, vol. 56, no. 3, pp. 508-519, 2013.

[26] N. Guo, J. Sun, H. Chen, H. Zhang, Z. Zhang, and D. Cai, "Liraglutide prevents diabetes progression in prediabetic OLETF rats," Endocrine Journal, vol. 60, no. 1, pp. 15-28, 2013.

[27] R. Hamilton, X. C. Thai, D. Ameri, and M. P. Pai, "Oral bioavailability of linezolid before and after Roux-en-Y gastric bypass surgery: is dose modification necessary in obese subjects?" Journal of Antimicrobial Chemotherapy, vol. 68, no. 3, pp. 666673, 2013. 


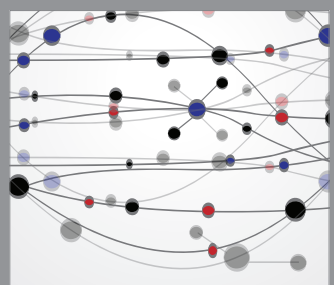

The Scientific World Journal
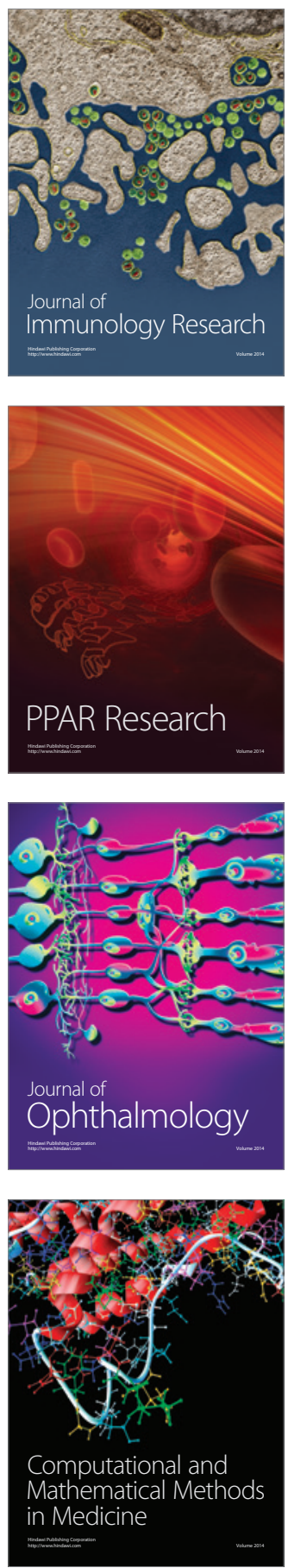

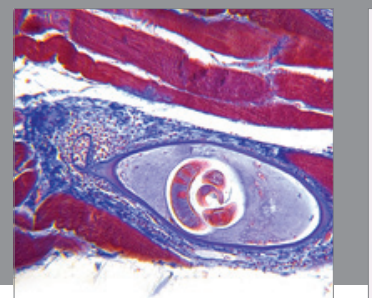

Gastroenterology

Research and Practice
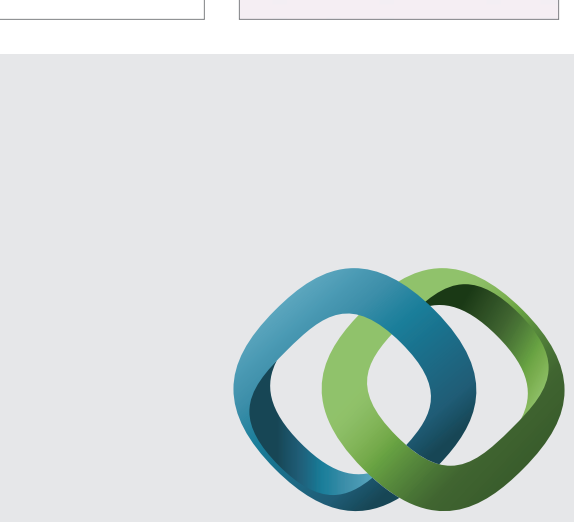

\section{Hindawi}

Submit your manuscripts at

http://www.hindawi.com
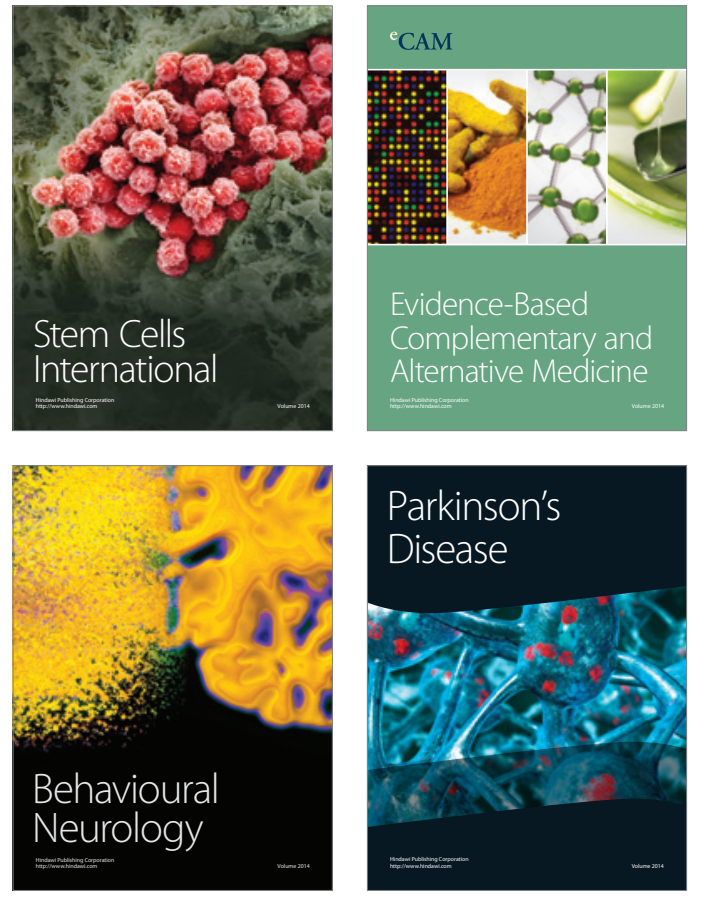
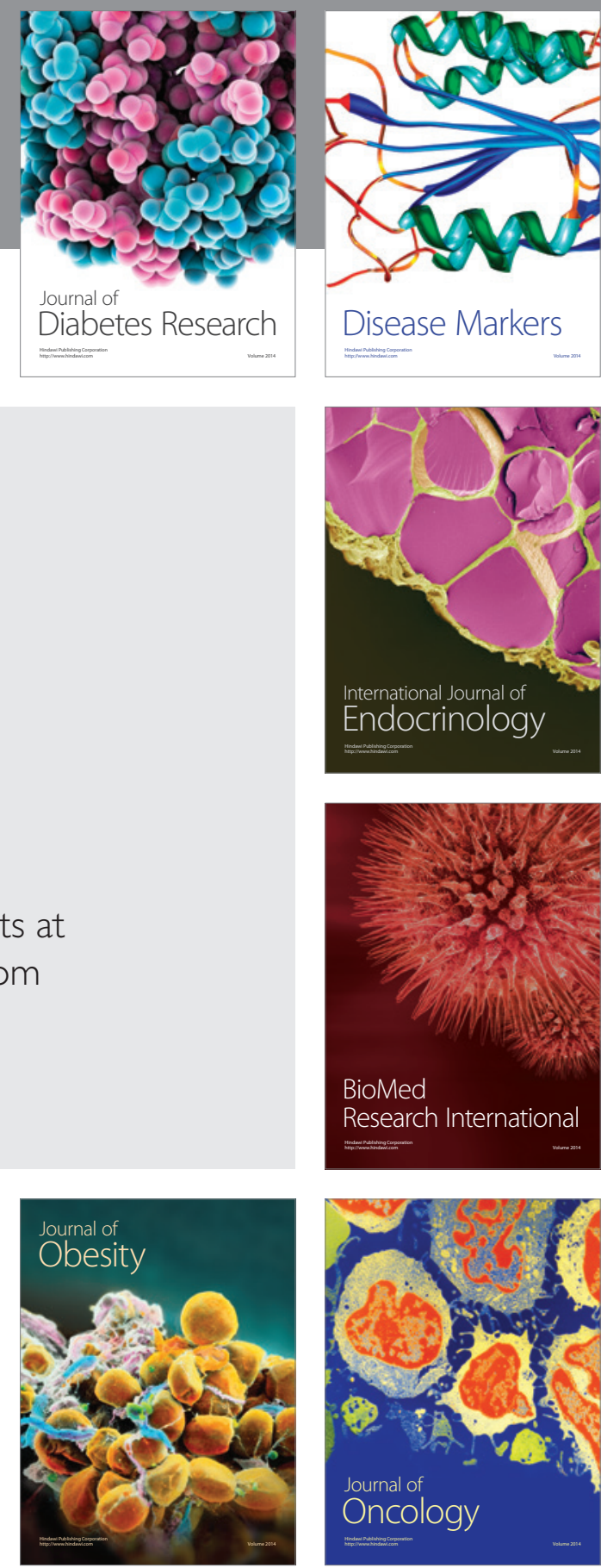

Disease Markers
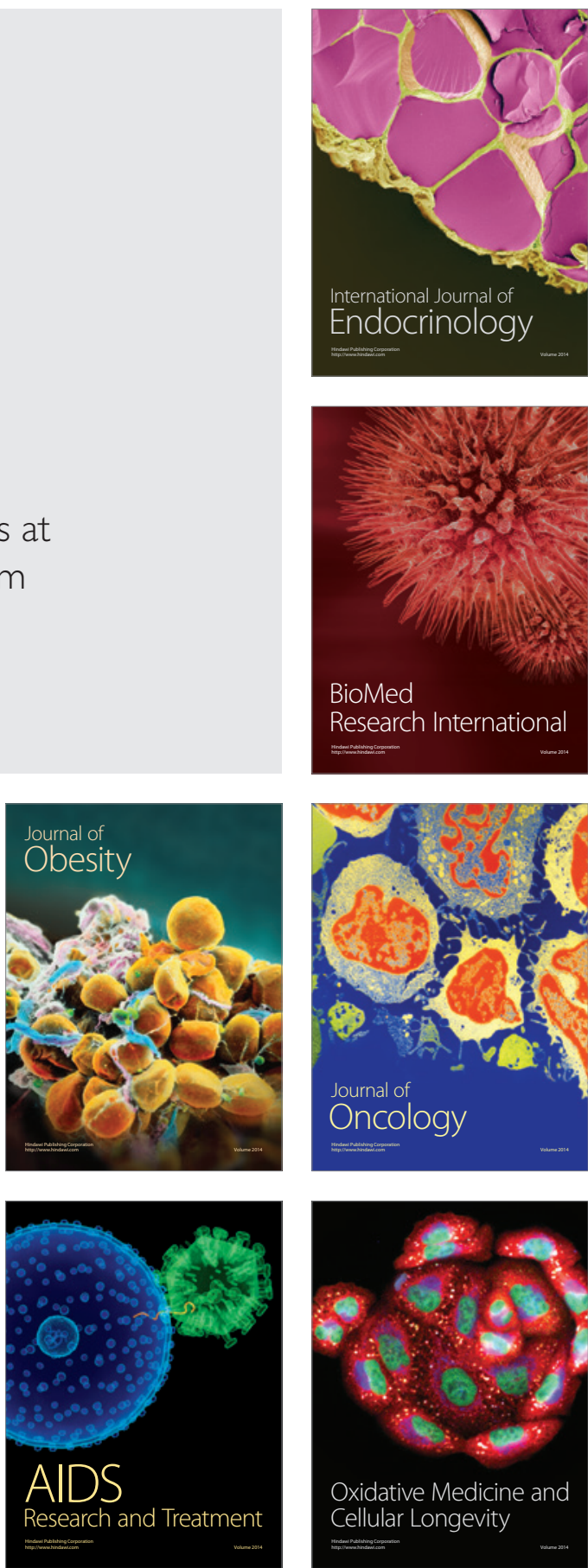